\title{
Inflationary Finance and the Welfare Cost of Inflation
}

\section{Citation}

Barro, Robert J. 1972. Inflationary finance and the welfare cost of inflation. Journal of Political Economy 80(5): 978-1001.

\section{Published Version}

doi:10.1086/259946

\section{Permanent link}

http://nrs.harvard.edu/urn-3:HUL.InstRepos:3451393

\section{Terms of Use}

This article was downloaded from Harvard University's DASH repository, and is made available under the terms and conditions applicable to Other Posted Material, as set forth at http:// nrs.harvard.edu/urn-3:HUL.InstRepos:dash.current.terms-of-use\#LAA

\section{Share Your Story}

The Harvard community has made this article openly available.

Please share how this access benefits you. Submit a story.

Accessibility 


\title{
Inflationary Finance and the Welfare Cost of Inflation
}

\author{
Robert J. Barro
}

Brown University and the University of Chicago

\begin{abstract}
This paper applies previous theoretical and empirical results on inflation and demand for money to a study of inflationary finance and the welfare cost of inflation. The amount of revenue generated by a steady inflation is derived as a function of the inflation rate and some underlying parameters. Empirically, the revenue-maximizing rate is on the order of 140 percent per month with the corresponding revenue approximating 15 percent of national income. It is argued that hyperinflations become unstable when the revenue-maximizing rate is exceeded. Because inflation leads to higher transaction costs (resulting from greater payment frequencies and reduced use of "money" as a payments medium), there is a net social cost attached to inflationary finance. The model implies that marginal collection costs of inflationary finance exceed 50 percent for all positive rates of inflation-hence, alternative means of raising revenue should be socially preferable. The analysis also provides estimates of the social gain from moving to the optimum quantity of money as 1-3 percent of income.
\end{abstract}

In a previous paper (Barro 1970b) I analyzed the impact of inflation on the demand for money. That paper developed a theoretical model which centered on the frequency of payments and the fraction of monetized transactions. The theory was applied to a study of demand for money during several hyperinflations. Section I of the current paper contains a brief summary of the earlier results. The remainder of the paper describes some theoretical and empirical extensions of these results.

In Section II monetary expansion is viewed as a vehicle for generating government revenue, and the earlier theory and empirical findings are utilized to relate the volume of inflationary finance to the (steady) mone-

National Science Foundation grant GS-3246 supported this research. I am grateful to Ed Feige, whose comments on a seemingly unrelated topic caused me to revise Section III of this paper. 
tary growth rate. The rate which maximizes steady-state revenue is also derived. Extreme rates from observed hyperinflations are compared with the revenue-maximizing rates in the context of stability of hyperinflation. It is argued that hyperinflations tend to become unstable (from the moneysupply side) when the revenue-maximizing rate is exceeded.

Section III considers the welfare cost or excess burden of anticipated inflation. The cost of inflation to individuals exceeds the volume of inflationary finance because increased inflation leads to increased transaction costs. In this model the increased transaction costs take two forms; first, an increased frequency of transactions (higher velocity) for those transactions which use money, and, second, an increase in the proportion of transactions which employ an alternative, but socially less efficient, medium of exchange. The analysis provides quantitative measures of welfare cost and marginal collection cost (welfare cost relative to revenue generation) as a function of the inflation rate. The results also provide an estimate of the potential social gain from achieving the optimum quantity of money.

Section IV contains some additional empirical results on hyperinflations. This section includes a comparison of the hyperinflationary experience in post-World War II Hungary with the post-World War I experience in the same country, and a test for the absence of money illusion in the demand for money during hyperinflations.

\section{Summary of Previous Results}

In the earlier model a real-income stream $Y / P$ is associated with an endogenous payment interval, $T / n$, where $n$ is the number of payments that occur over a time interval $T$. It is assumed that the real cost of making (wage) payments is solely a lump-sum amount $a / P$. Prices change at the constant (proportionate) rate $r_{p}$. The real rate at which increases in the payments period are discounted is $r^{*}$. In the case of deferred wage payment, a lengthening of the pay period implies an increase in the average loan outstanding from employees to employers (Barro 1970b, p. 1235). The appropriate discount rate for the payments period is therefore the difference between the rates imputed by employees and employers, $r^{*}=r_{h}-r_{f}$, where $r_{h}$ is the employee (household) rate and $r_{f}$ is the employer (firm) rate. In the earlier analysis $r_{f}$ was assumed to be (approximately) zero; hence $r^{*}$ was equal to $r_{h}$.

If all payments corresponding to the income stream $Y / P$ are monetized, the cost per time (to employers and employees) associated with the period $T / n$ can be approximated by ${ }^{1}$

${ }^{1}$ Equation (1) assumes that employers receive income and employees spend income at a uniform rate, and that no satisfactory intermediate assets are available-that is, both units hold money until either a wage payment or a purchase of commodities 


$$
Z \approx\left(r_{p}+r^{*}\right)(M / P)+\frac{a}{P} \frac{n}{T} \approx\left(r_{p}+r^{*}\right) \frac{Y}{P} \frac{T}{n}+\frac{a}{P} \frac{n}{T} .
$$

The first term in the cost expression corresponds to the interest cost on average (employer plus employee) money balances: $(M / P) \approx(Y / P)$ $(T / n)$. The second term describes the cost of making payments at the rate $n / T$. The cost-minimizing payments period is

$$
T / n=\sqrt{\frac{a / P}{Y / P\left(r_{p}+r^{*}\right)}} .
$$

As inflation intensifies, the optimal pay period declines in equation (2), thus signifying a direct increase in velocity and a corresponding reduction in real money holding.

Denoting the endogenously determined fraction of transactions which employ money by $(1-\Phi)$, the average holding of money is given by (using eq. [2])

$$
\frac{M}{P}=(1-\Phi) \frac{Y}{P} \frac{T}{n}=\frac{(1-\Phi) A(Y / P)}{\sqrt{r_{p}+r^{*}}},
$$

where $A=\sqrt{(a / P) /(Y / P)}$ is assumed to be constant (since $a / P$ is regarded as primarily an income-foregone cost). In equation (3), if the monetization fraction, $(1-\Phi)$, is fixed the elasticity of money demand with respect to the inflation rate approaches $-1 / 2$ as $r_{p}$ becomes large relative to $r^{*}$. For moderate rates of (positive) inflation, the (absolute) elasticity is kelow one-half.

From the previous analysis, the determination of $\Phi$ involves a weighing of the inflationary cost attached to the continued use of money against some loss of convenience (increased transaction costs) associated with a switch to an alternative, relatively stable-valued transactions medium (such as barter). Under a particular assumption concerning the distribution over types of transactions of the benefit of money usage (a gamma distribution), the fraction of monetized expenditures can be written as an explicit function of the inflation rate: ${ }^{2}$

occurs. The formula also involves a minor approximation of the form $\left(r_{p}+r^{*}\right) T / n$ $\ll 1$.

2 The earlier paper contains some errors in the derivation of $\Phi$ (Barro 1970b, p. 1241). If, for the $i^{\text {th }}$ group of transactions, $\Phi_{i}$ denotes the percentage of nonmonetized transactions, $(Y / P)_{i}$ denotes the total volume of transactions, and $(\Psi / P)_{i}$ measures the transactions benefit of employing money instead of the substitute medium per amount of monetized transaction (where the groupings over $i$ are determined so that $[\psi / P]_{i}$ is constant), then eq. (30) in the previous paper indicated that $\Phi_{i}$ should be chosen to maximize $R_{i}=\left(1-\Phi_{i}\right)(Y / P)_{i}(\psi / P)_{i}-2 A\left(1-\Phi_{i}\right)(Y / P)_{i}\left(\sqrt{r_{p}+r^{*}}-\right.$ $\left.\sqrt{r^{*}}\right)$. The correct criterion is to maximize $R_{i}=\left(1-\Phi_{i}\right)(Y / P)_{i}(\Psi / P)_{i}-2 A(Y / P)_{i}$ $\left[\sqrt{\left(1-\Phi_{i}\right)\left(r_{p}+r^{*}\right)}+\sqrt{\Phi_{i}\left(r^{*}\right)}\right]$. However, the two criteria lead to the identical corner solution for choosing $\Phi_{i}$, namely, $\Phi_{i}=1$ if $(\psi / P)_{i}<2 A\left(\sqrt{r_{p}+r^{*}}-\sqrt{r^{*}}\right)$; 


$$
\begin{array}{rr}
(1-\Phi)=\left[1+k\left(\sqrt{r_{p}+r^{*}}-\sqrt{r^{*}}\right)\right] & \\
\cdot \exp \left[-k\left(\sqrt{r_{p}+r^{*}}-\sqrt{r^{*}}\right)\right] & \left(r_{p} \geqslant 0\right) \\
(1-\Phi)=1 & \left(r_{p} \leqslant 0\right)
\end{array}
$$

where "exp" indicates exponentiation from base $e$. In equation (4) the fraction of monetized transactions is inversely related to the inflation rate, with the magnitude of the (negative) response determined by the nonnegative parameter $k$. In the theoretical model the $k$ parameter is inversely related to the average cost per amount of transaction of employing a money-substitute transactions medium.

Combining equations (3) and (4) yields a demand-for-money function in which the inflation-rate elasticity can be approximated as

$$
\eta_{r_{p}} \approx-\frac{1}{2}\left(\frac{r_{p}}{r_{p}+r^{*}}+\frac{k^{2} r_{p}}{1+k \sqrt{r_{p}}}\right)
$$

For moderate values of $r_{p}\left(r_{p} \sim r^{*}\right)$, the term involving $k$ is empirically negligible and $\eta_{r_{p}}$ can be approximated by the first term on the right side of equation (5). For very high values of $r_{p}$, the second term, which derives from the substitution of an alternative transactions medium, becomes important. Since $k>0$, the inflation-rate elasticity eventually rises (absolutely) above one-half. The rate of increase in the absolute elasticity is positively related to $k$ and, therefore, inversely related to the average cost of employing the alternative payments medium.

The model described above was extended to situations where the inflation rate varied over time through the development of an effective rate-ofinflation $\left(\pi^{e}\right)$ mechanism. Essentially, a distributed-lag mechanism involving a variable coefficient of adjustment was derived. The discrete form of the distributed-lag model is (when $r^{*} \ll \pi^{e}$ )

$$
\begin{gathered}
\sqrt{\pi_{t}}=\beta_{t} \sqrt{\pi_{t}}+\left(1-\beta_{t}\right){\sqrt{\pi_{t-1}}}^{e} \\
\beta_{t}=1-\exp \left[-b\left(\bar{\pi}_{t}^{e}\right)^{\frac{3}{4}}\left(\frac{1}{1-\bar{\Phi}_{t}}\right)\right](b>0),
\end{gathered}
$$

\footnotetext{
$\Phi_{i}=0$ otherwise. Therefore, no substantive change from the previous result is required. The solution for $(1-\Phi)$ indicated in eq. (4) follows from the assumption that $(\psi / P)_{i}$ is distributed, independently of $(Y / P)_{i}$, according to a second-order gamma distribution. The formula in eq. (3), $M / P=(1-\Phi)(Y / P)(T / n)$, and not $M / P=\sqrt{1-\Phi}$ $(Y / P)(T / n)$, is correct if $(1-\Phi)$ is interpreted as the fraction of economic units (as well as the fraction of transactions) which employ money. I am grateful to Gary Gillum for pointing out the errors in the previous analysis.
} 
where $\pi_{t}$ is the actual (average) rate of inflation between $t-1$ and $t$, a bar indicates an average of $t$ and $t-1$ values, and $b$ is an unknown coefficient, with $\left(\partial \beta_{t} / \partial b\right)>0,\left(\partial \beta_{t} / \partial \bar{\pi}_{t}{ }^{e}\right)>0$, and $0<\beta_{t}<1$.

Given a value for $b$ and a time series for $\pi$ (and a starting point for $\pi^{e}$ ), equation (6) determines a time series for $\pi^{e}$. The $\pi^{e}$ variable is then incorporated into the money-demand model of equations (3) and (4):

$$
\begin{gathered}
\log (M / P)=\alpha_{1}+\alpha_{2} \log \left(\pi^{e}+r^{*}\right)+\log (1-\Phi) ; \\
(1-\Phi)=\left[1+k\left(\sqrt{\pi^{e}+r^{*}}-\sqrt{r^{*}}\right)\right] \\
\cdot \exp \left[-k\left(\sqrt{\pi^{e}+r^{*}}-\sqrt{r^{*}}\right)\right],
\end{gathered}
$$

where the theory suggests: $\alpha_{2}=-1 / 2, k>0$.

Making the approximations: $\pi^{e}>>r^{*} \approx 0$ and $\alpha_{1} \approx \log (A \cdot Y / P) \approx$ constant, the four parameters $\left(\alpha_{1}, \alpha_{2}, k, b\right)$ of equations (6) and (7) were previously estimated for four cases of post-World War I hyperinflation-Austria, Germany, Hungary (Hungary I), and Poland (Barro $1970 b$, p. 1255). Results for post-World War II Hungary (Hungary II) have now been obtained in the same framework and are included in table 4. These results will be discussed in detail in Section IV. Since the underlying theory suggests $\alpha_{2}=-1 / 2$, and since this conjecture was borne out by the empirical results for the five cases of hyperinflation, estimates of $\left(\alpha_{1}, k, b\right)$ have also been obtained subject to the constraint: $\alpha_{2}=-1 / 2$. These estimates are included in table 3 and are used in the discussion of Sections II and III.

\section{Inflationary Finance}

This section is concerned with the volume of steady-state inflationary finance associated with a constant monetary growth rate, $\mu=(1 / M)$ $(d M / d t)$. In order to derive steady-state properties within the simple monetary framework for which empirical results are available, certain simplifying assumptions are necessary. Specifically, it is assumed that the total volume of real transactions, $Y / P,{ }^{3}$ and the real rate of discount, $r^{*}$, are independent of the (steady) growth rate of the money stock, $\mu$. Essentially, all variations in time spent conducting transactions (reflected in changed velocity and changed fraction of monetized transactions) are assumed to come out of leisure. Further, any real-balance-type effects on $r^{*}$ are neglected. ${ }^{4}$ If the above assumptions are violated, the subsequent

${ }^{3}$ In the theoretical model, $Y / P$ corresponds to income, rather than to total, transactions. The important assumption is that total transactions remain fixed. See n. 6, below.

4 A discussion of the leisure assumption is contained in Bailey (1956, p. 102). The general issue of the effect of the long-term monetary growth rate on real rates of return is summarized in Stein (1970). However, it should be recalled that $r^{*}$ corre- 
analysis does not lose validity. However, if $Y / P$ and $r^{*}$ are endogenous, additional technical and behavioral relationships must be brought into the model. As far as $Y / P$ and $r^{*}$ can be satisfactorily treated as exogenous (that is, independent of $\mu$ ), the need to examine these additional relationships (which have, in any case, not been investigated empirically for the cases under consideration) is removed.

In addition to the above assumptions, real growth is abstracted from (population and technology fixed), and use is made of the steady-state equality between the monetary growth rate and the inflation rate: $\mu=r_{p}$. Given this steady-state condition, the real amount of inflationary finance can be written as ${ }^{5}$

$$
G=\frac{d M / d t}{P}=\mu \frac{M}{P}=r_{p} \cdot \frac{M}{P}\left(r_{p}\right) .
$$

Using the expression for $(M / P)\left(r_{p}\right)$ from equation (3) and substituting for $(1-\Phi)$ from equation (4) yields an explicit expression for $G$ in terms of $r_{p}$ :

$$
\begin{aligned}
G=A(Y / P) \frac{r_{p}}{\sqrt{r_{p}+r^{*}}}\left[1+k\left(\sqrt{r_{p}+r^{*}}-\sqrt{r^{*}}\right)\right] \\
\cdot \exp \left[-k\left(\sqrt{r_{p}+r^{*}}-\sqrt{r^{*}}\right)\right] .
\end{aligned}
$$

The empirical results in table 3 provide estimates from five cases of hyperinflation of $A(Y / P)$ and $k$. Since the $A(Y / P)$ values depend on arbitrary index specifications, it is of greater interest to consider the volume of government revenue as a fraction of some measure of aggregate income. An approximate real income index is constructed for each case by using velocity data contained in Bailey's study (1956, p. 99, table 2, col. 5). The details of the construction of these indices are contained in table 1. If the real income measure is denoted by $X, g=G / X$ is determined by $[A(Y / P)] / X$ in equation (9). ${ }^{6}$ Estimates of $[A(Y / P)] / X$, indicated for each case of hyperinflation in table 1 , are independent of arbitrary index base specifications.

In the empirical estimation the real rate of discount, $r^{*}$, was assumed

sponds to the difference between two real rates of return, $r_{h}-r_{f}$, rather than to the absolute return, which would generally be identified with $r_{f}$.

5 If the government issues only some portion of the total money supply, the finance is shared with other issuers of money. However, a full analysis would also have to consider the state of competition in the banking industry.

${ }^{6}$ If $X$ and $Y / P$ were equivalent, $[A(Y / P)] X$ would be an estimate of $A=\sqrt{(a / P) /}$ $\overline{(Y / P)}$. The key problem in using this estimate is the distinction between income transactions and total transactions. In the original model the flow of final (real income) payments, $Y / P$, was associated with two transaction flows (sales and factor payments). To the extent that additional intermediate transactions occur, $A(Y / P)$ refers to this larger volume of transactions; hence, $[A(Y / P)] X$ is an estimate of $A$ multiplied by the ratio of total transactions to (twice) income transactions. 


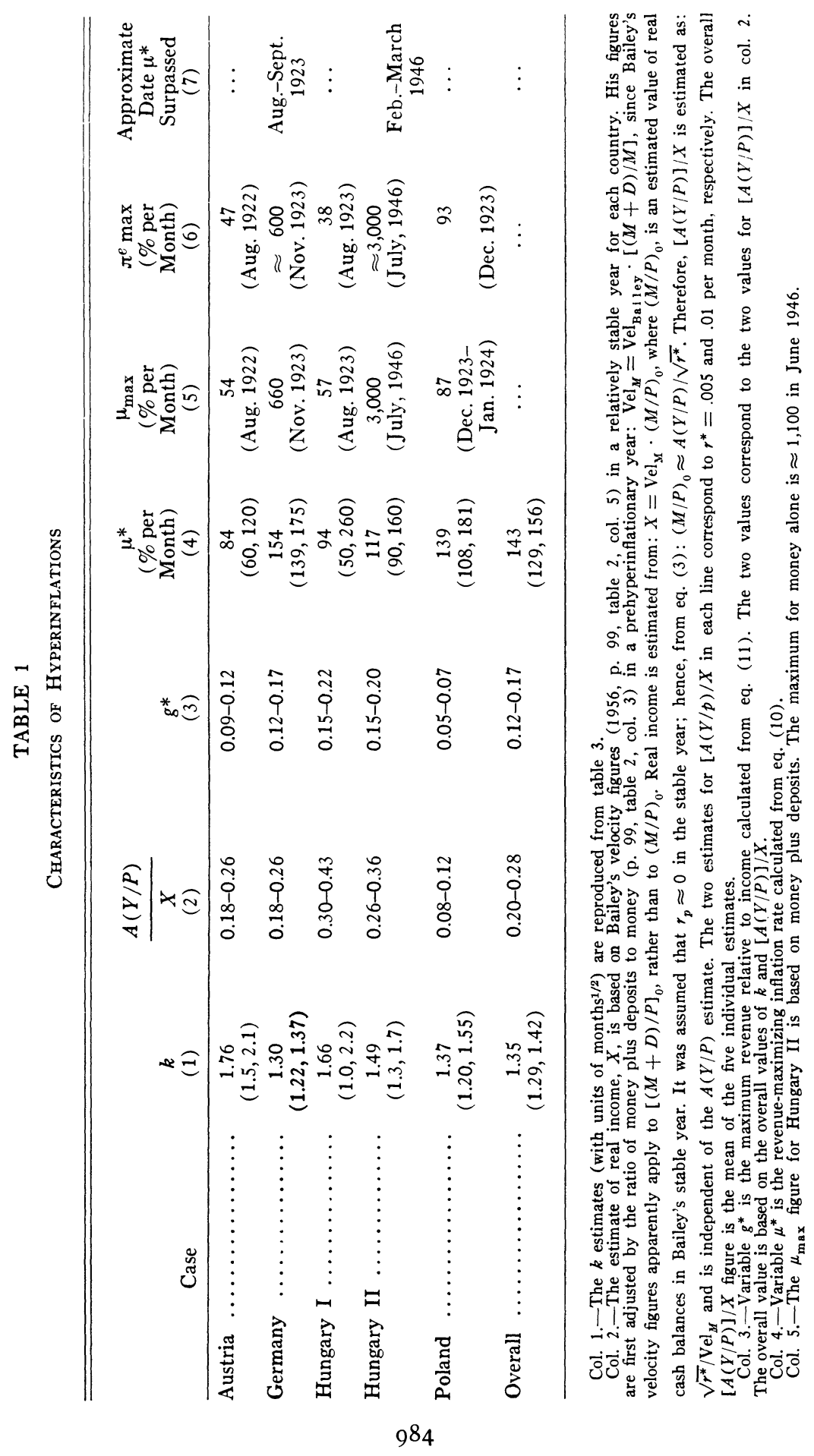


to be negligible relative to the extreme rates of inflation which prevailed. Therefore, $r^{*}$ was set equal to zero for each case and was not estimated from the data. However, if one is concerned with moderate rates of inflation, a nonzero value for $r^{*}$ is appropriate. Since direct estimates of $r^{*}$ are unavailable, the calculations are based on two values which appear to provide a plausible a priori range: $r^{*}=1 / 2$ percent per month and $r^{*}=1$ percent per month. ${ }^{7}$

A tabulation of $g$ versus $r_{p}$ is contained in table 2. The calculation of $g=G / X$ from equation (9) utilizes the overall parameter estimates of $[A(Y / P)] / X$ and $k$ from the five cases of hyperinflation (table 1) ${ }^{8}$

At a 6 percent annual rate of price change ( $1 / 2$ percent per month) government revenue is about 1 percent of total income. This magnitude should be illustrative for cases of moderate inflation, though some modification is needed to account for the effect of real growth (see n. 9, below). At an inflation rate of 5 percent per month (which characterizes the most extreme Latin American experiences), inflationary finance is between 4 and 6 percent of income. Inflationary finance reaches a peak of 13-19 percent of income at an inflation rate in the vicinity of 150 percent per month. This peak behavior has particular significance for the stability of hyperinflation and is discussed in detail below.

Since government revenue is determined by the inflation rate from equations (8) and (9), one may consider the monetary growth rate $\left(\mu=r_{p}\right)$ which maximizes the steady-state volume of inflationary finance. Since revenue is given by: $G=r_{p} \cdot(M / P)\left(r_{p}\right)$, the "optimal" expansion rate is the one which corresponds to a money-demand elasticity of -1 (see Cagan 1956, pp. 77-86).$^{9}$ In the current model the inflation-rate elasticity of money demand is determined from equation (5). If the effect of substitute transactions media is neglected $(k=0)$, the (absolute) elasticity never exceeds one-half. Therefore, if the conventional money is

${ }^{7}$ Friedman (1969, pp. 40, 41, 44) uses a similar concept, "internal rate of discount," which he estimates lies in the interval of $0.05-0.33$ per year. The values of $r^{*}$ used in this paper are on the low end of the range proposed by Friedman.

${ }^{8}$ In the earlier statistical study the estimated $k$ values did not differ significantly from each other. However, with $\hat{\alpha}_{2}=-1 / 2$ restricted in each case, the estimated $k$ values indicated in table 3 do differ significantly. The relevant statistic is $-2 \log \lambda=14.3$, where $\lambda$ is the likelihood ratio. The critical $\chi^{2}$ value for the null hypothesis of equal $k$ 's, $\chi^{2}(4){ }_{.05}=9.5$, is exceeded at the .05 level. Tests of equality for the $[A(Y / P)] / X$ parameters could not be performed, since they depended on Bailey's velocity estimates, which have unknown reliability.

9 This analysis must be modified in the presence of real growth. Friedman (1971) has shown that steady-state government revenue is given by: $G=\left(r_{p}+\rho\right)(M / P)$, where $\rho=r_{n}+\eta_{y} r_{y}, r_{n}$ is the proportionate growth rate of population, $r_{y}$ is the proportionate growth rate of real capita income, and $\eta_{y}$ is the elasticity of real per capita money demand with respect to real per capita income. The revenue-maximizing inflation rate is then determined from the condition $\eta_{r_{p}}=-1+\rho /\left(r_{p}+\rho\right)$. Hence, the absolute elasticity which corresponds to the maximum revenue is below one, when $\rho>0$. 


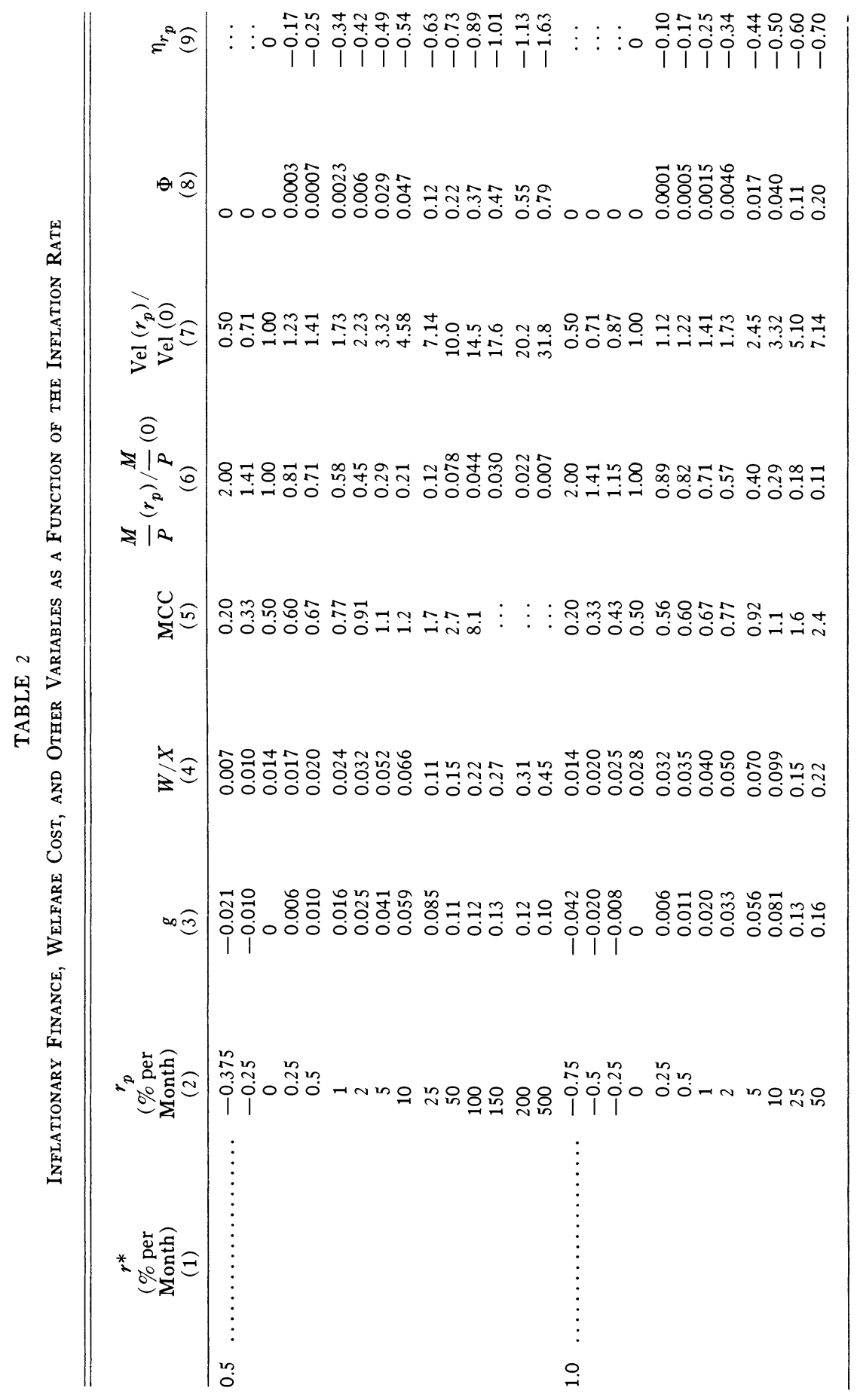




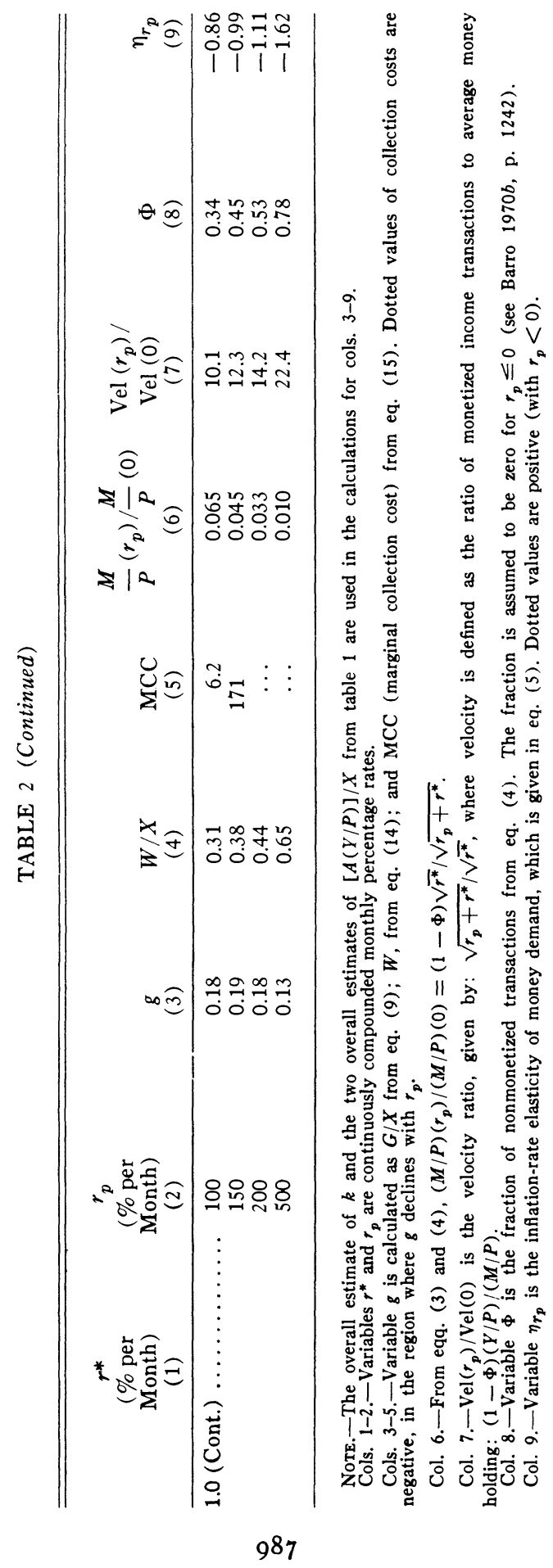


retained for all payments (and, unrealistically for very high rates of inflation, if the volume of payments remains constant), no bound would exist on the volume of inflationary finance. However, with the introduction of the money-substitute effect, the inflation-rate elasticity increases (absolutely) beyond one-half as the inflation rate rises. The inflation rate at which the elasticity reaches -1 can be determined explicitly as an inverse function of the money-substitute parameter $k$. The solution to a quadratic equation based on equation (5) yields an approximate relation for the revenue-maximizing rate:

$$
\mu^{*} \approx \frac{2.6}{k^{2}} \quad\left(r^{*} \ll \mu^{*}\right) .
$$

Therefore, a small value of $k$ (high average cost for employing substitute transactions media) corresponds to a high revenue-maximizing rate of monetary expansion.

The amount of revenue relative to total real income at the rate $\mu^{*}$ can be explicitly calculated from equation (9) as

$$
g^{*} \approx \frac{0.84}{k} \frac{A(Y / P)}{X} .
$$

Values of $\mu^{*}$ and $g^{*}$ are indicated in table 1 for the five individual cases of hyperinflation and for the parameters corresponding to the overall sample. The values of $k$ and $[A(Y / P)] / X$ are the empirical estimates indicated in table 1 . The estimated values of $\mu^{*}$ range from about 80 percent per month for Austria to about 150 percent per month for Germany. ${ }^{10}$

10 In Cagan's study (1956, p. 81, table 9, col. 1) the estimated revenue-maximizing rates are much lower than those derived in the current analysis. His range is about 11 percent per month for Austria and Hungary I to 44 percent per month for Poland. Cagan notes (p. 81) that "the actual rates were well above the constant rates that would have maximized the ultimate revenue." For the $\mu^{*}$ values calculated in this paper (table 1), this conclusion holds for only two of the five cases considered. A likely explanation for the divergence between Cagan's results and mine is that Cagan's demand-for-money function, $M / P=e^{--(a r p+\gamma)}$, has the inflation-rate elasticity, $\eta_{r_{p}}=-\alpha r_{p}$, in which the elasticity increases (absolutely) directly with $r_{p}$. In the model used in this paper (eq. [5]) the elasticity increases with $r_{p}$ at a slower rate. Therefore, the rate at which the elasticity equals -1 is reached at a lower inflation rate with Cagan's function. (This conclusion actually depends on the fact that the parameters are estimated from a sample where the average value of $r_{p}$ is much below the revenue-maximizing rate.) Bailey's numerical results are based on the moneydemand functions which Cagan estimated. The relative empirical merits of Cagan's money-demand function and the function used in the current paper are discussed in Barro (1970b, pp. 1257-58) for the four post-World War I cases. The results in Cagan's form for the second Hungarian case are contained in table 4 . In a recent study Friedman (1971) estimates revenue-maximizing rates of inflation which are below 20 percent per year. Hence, his estimates are far belov those given by Cagan, which were, in turn, below those presented here. The explanation for Friedman's result is threefold: First, he uses Cagan's form of money-demand function, which, as indicated above, tends to produce lower estimates of the revenue-maximizing rate. Second, he uses 
The estimates of maximum revenue range from 5-7 percent of national income for Poland to 15-22 percent for Hungary I. Corresponding to the overall estimate of $k$, the overall estimate of $\mu^{*}$ is about 140 percent per month, which corresponds to $12-17$ percent of national income. ${ }^{11}$

Table 1 also contains values of $\mu_{\max }$, the actual maximum of monthly average monetary growth rate which occurred during each hyperinflation, and $\pi_{\max }{ }^{e}$, the extreme value of the effective rate of inflation which occurred. Since steady-state measures are involved, it may be useful to compare the estimated value of $\mu^{*}$ with $\pi_{\max }{ }^{e}$, as well as with $\mu_{\max }$, in order to determine whether the optimum expansion rate was exceeded (for a significant time period) in each case. One may conclude from table 1 that the revenue-maximizing rate was exceeded for Germany and Hungary II but not for Austria, Hungary I, and Poland.

\section{The Stability of Hyperinflation}

The view of monetary expansion as a vehicle for generating government revenue may be used to analyze one aspect of the stability of hyperinflation. If an economy is initially in a noninflationary situation, government revenue may be generated by instituting a positive value of $\mu$. Additional expenditures may be financed by further increases in $\mu$, and each increase is successful (that is, actually results in a higher steady-state revenue) as long as $\mu<\mu^{*}$. However, if a total steady-state revenue in excess of $G^{*}=$ $g^{*} \cdot X$ is desired, inflationary finance cannot suffice. When $\mu$ is pressed beyond $\mu^{*}$, revenue declines (at least, eventually) rather than rises. If the government is oblivious to the basic problem, the reaction to this reduced revenue may be an additional increase in $\mu$, which produces further reductions in revenue and creates added pressure for even higher rates of expansion. Eventually, a nearly complete flight from money would result, and the "benefit" of inflationary finance would vanish. At this point (or possibly sooner if the government is astute), considerable pressure can be expected for basic monetary reform (which often involves renaming the currency unit but, in its essence, involves curtailing the growth rate of the money stock).

From an empirical standpoint, the five cases of hyperinflation tend to substantiate this sort of process. The most interesting evidence derives

estimates of the $\alpha$-coefficient in Cagan's form (see above) which are ten to twenty times as large as those estimated by Cagan, thus reducing the estimated revenuemaximizing inflation rate by a factor of ten to twenty. Third, he includes a nonzero value for real growth ( $\rho$ in n. 9, above) which also tends to lower the estimated rate. Friedman stresses this inclusion of real growth as the explanation for his low estimates. However, if real growth had been included in the money-demand function of the current study, the impact on the estimates of $\mu *$ in table 1 would have been negligible.

11 Since these values are calculated from eqq. (10) and (11), they are approximations which neglect $r^{*}$ relative to $\mu^{*}$. For example, with $r^{*}=1$ percent per month, the maximum $g^{*}$ value in table 2 is 19 percent rather than 17 percent. 
from the German case, in which the inflationary course appeared quite stable for a number of years, despite values of $\mu$ and $\pi^{e}$ in excess of 60 percent per month (Barro $1970 b$, table 2 ). After reaching $\mu=0.66 /$ month $\left(\pi^{e}=0.63\right)$ in February 1923, the inflation receded for several months and reached $\mu=0.20 /$ month $\left(\pi^{e}=0.20\right)$ in May 1923. The February rate of expansion was not attained again until July $\left(\mu=0.88, \pi^{e}=0.71\right.$ in July). When the critical monetary expansion rate, $\mu^{*}=1.5[1.4,1.7]$ per month, was exceeded in August-September $1923\left(\mu=1.5, \pi^{e}=2.7\right.$ in August; $\mu=3.4, \pi^{e}=3.1$ in September), the inflation went completely out of control. The explosive process was not contained until the sweeping monetary reform of November 1923.

A similar analysis is applicable to the last months of the second Hungarian hyperinflation. The critical monetary expansion rate, $\mu^{*}=1.2$ $[0.9,1.6]$ per month, was first approached in November $1945(\mu=1.2$, $\pi^{e}=1.0$ in November). The process did not explode at this point, since $\mu$ dropped below 0.8 per month in December 1945 and January $1946\left(\pi^{e}\right.$ $=0.92$ and 0.75 , respectively). However, the critical expansion rate was definitely exceeded in February-March $1946-\mu=1.3, \pi^{e}=1.7$ in February; $\mu=1.9, \pi^{e}=1.4$ in March. The inflation went entirely out of control between April and July 1946, with the maximum rates of inflation dwarfing those of the German experience. The process was finally terminated in a monetary reform at the end of July 1946.

The Austrian and the first Hungarian cases both involve the confinement of actual rates of monetary expansion below the critical rate, with no obvious appearances of instability. For Austria, the peak monetary expansion was 0.54 /month in August-September $1922\left(\pi^{\mathrm{e}}=0.47\right.$ and 0.31 , respectively), which was well below $\mu^{*}=0.8[0.6,1.2]$ per month. After September, $\mu$ and $\pi^{e}$ declined gradually, reaching $\mu=0.18, \pi^{e}=$ 0.10 in December. In $1923, \mu$ varied between 0.12 and zero per month, while $\pi^{e}$ varied between 0.08 and 0.04 per month. For Hungary I, the peak expansion occurred in August 1923 with $\mu=0.57$ per month $\left(\pi^{e}=\right.$ 0.38 ), which was below $\mu^{*}=0.9[0.5,2.6]$ per month. Subsequently, there was a gradual decline in $\mu$, although the rate of expansion remained above 0.10 per month until November 1924.

For Poland, the evidence is less clear. The peak expansion occurred during the four-month period of October 1923 to January 1924, during which $\mu$ was between 0.62 and 0.87 per month, and $\pi^{e}$ was between 0.74 and 0.93 per month. These rates of expansion were close to, but below, the critical rate: $\mu^{*}=1.4[1.1,1.8]$ per month. The behavior subsequent to the peak expansion involved a rapid reduction in $\mu$ and $\pi^{e}$, reaching $\mu=$ 0.04 per month and $\pi^{e}=0.13$ per month in April 1924. Although the Polish experience did not exhibit the obvious instability which marked the German and second Hungarian cases, an extensive monetary reform did occur in Poland in April 1924. 


\section{The Welfare Cost of Inflation}

This section considers the net welfare cost, or excess burden, imposed on an economy by a steady, anticipated rate of inflation. ${ }^{12}$

The revenue obtained by government, $\mu(M / P)$, is directly balanced by an inflationary cost to individuals, $r_{p}(M / P) \cdot{ }^{13}$ The net welfare cost of inflation (abstracting from any direct costs of printing money) derives from the transaction costs which individuals incur in order to avoid the private interest costs associated with holding money. In the current model increases in inflation induce increases in transaction costs in two respects: first, individuals raise the frequency of transactions (increase velocity) for monetized transactions, and, second, individuals employ alternative payments media with higher transaction costs for a greater proportion of their transactions. At moderate rates of inflation, the substitution of alternative payments media for money is empirically unimportant. It is shown in table 2 that $\Phi$, the fraction of transactions conducted via a substitute media, remains below 1 percent until $r_{p}$ reaches a value between 2 and 5 percent per month-that is, until the rate of inflation becomes substantially larger than $r^{*}$. Hence, for inflation rates with magnitudes equal to or below the order of $r^{*}$, changes in transaction costs produced by changes in $r_{p}$ reflect mostly alterations in the frequency of monetized transactions. In this range of $r_{p}$ the total transaction costs can be approximated by $(a / P)(n / T)$, which is the cost of conducting monetized transactions at frequency $n / T$ in equation (1). Moreover, when the payments period is selected according to the optimal policy indicated in equation (2), individuals act so as to equate the (total) amount of transaction costs to the (total) amount of interest costs. ${ }^{14}$ That is,

$$
\begin{aligned}
W \approx(a / P)(n / T)=\left(r_{p}+r^{*}\right) & (M / P) \\
& \approx A(Y / P) \sqrt{r_{p}+r^{*}}\left(\text { when } r_{p} \sim r^{*}\right),
\end{aligned}
$$

12 This analysis follows Bailey (1956, pp. 93, 94) in abstracting from costs which involve the uncertainty of the inflationary course. In particular, distribution effects are ignored. It is further assumed that open, rather than suppressed, inflation is occurring.

${ }^{13}$ Since the total interest cost in eq. (1) is $\left(r_{p}+r^{*}\right)(M / P)$, the $r^{*}(M / P)$ portion is not offset by the flow of government revenue. However, an increase in $M / P$ can be viewed as an increase in private real wealth which is evaluated in flow terms by individuals as $r *(M / P)$. Hence, the increase in real wealth offsets the real interest-foregone cost, $r^{*}(M / P)$, in eq. (1). However, to the extent that a change in $M / P$ reflects a change in the average holding of goods inventories, the change in real interest foregone on these goods holdings would be an element of both private and social cost. This aspect was not treated in my earlier model, since a uniform pattern of expenditures was assumed (Barro 1970b, p. 1237), but has been considered in some recent theoretical work (Clower 1969; Feige and Parkin 1971; Santomero 1971). See also n. 16, below.

14 The condition for the optimal $T / n$ is that the marginal reduction in transaction costs just balance the marginal increment in interest costs. However, the value of $T / n$ chosen in this manner (eq. [2]) is also such as to equate the total amount of transaction costs to the total amount of interest costs in eq. (1). 
where $W$ is the welfare cost, which corresponds to the resources used up in undertaking monetized transactions at frequency $n / T$.

The minimum welfare cost in equation (12) is zero, which is attained at $r_{p}=-r^{*}$. That is, the welfare cost disappears when money bears real interest at rate $r^{*}$. At this point, the private cost which individuals attach to holding money, which depends on the rate $r_{p}+r^{*}$, is zero-hence, individuals are not mntivated to engage in costly transactions in order to economize on private interest costs. Since, in fact, there is no social cost attached to holding money (that is, the interest cost is a private, but not a social, cost), while there is a social cost attached to making transactions, the condition $r_{p}=-r^{*}$ leads to the social optimum where welfare cost disappears. ${ }^{15}$ This conclusion corresponds to the usual result in the literature on the optimum quantity of money-see, for example, Friedman (1969, pp. 33-34).

Since $\Phi \approx 0$ when $r_{p} \sim r^{*}$, government revenue is determined from equation (3) as

$$
G=r_{p}(M / P) \approx \frac{A(Y / P) r_{p}}{\sqrt{r_{p}+r^{*}}}\left(\text { when } r_{p} \sim r^{*}\right) .
$$

Hence, the marginal collection cost (MCC) of inflationary finance is, using equation (12),

$$
\mathrm{MCC}=d W / d G \approx \frac{r_{p}+r^{*}}{r_{p}+2 r^{*}}\left(\text { when } r_{p} \sim r^{*}\right) .
$$

The MCC is zero at $r_{p}=-r^{*}$, rises to one-half at $r_{p}=0$, and rises above one-half as $r_{p}$ becomes positive. The important result is that the marginal collection cost of inflationary finance exceeds one-half for all positive rates of inflation.

For very high rates of inflation $\left(r_{p}>2-5\right.$ percent per month), the substitution of alternative payments media becomes important, and equation (13), which omits this substitution possibility, will significantly understate the marginal collection cost of inflationary finance. The welfare cost must now include the transaction costs associated with the use of (socially) less efficient payments media, along with the cost of conducting monetized transactions, which has already been considered. ${ }^{16}$ Both com-

\footnotetext{
15 In effect, since transactions are costly and serve no social function, transactions do not occur at the social optimum. Frankly, I find this result disturbing, although it does seem to agree with the conventional optimum-quantity-of-money result. Perhaps some new conclusions would emerge from a model where transactions served a social function in the sense of providing information and/or economizing on search activity.

16 The welfare cost must also include the real interest cost attached to the average holding of the alternative payments media. That is, the real interest foregone on holdings of such alternative payments media as physical commodities and foreign exchange constitutes a net social cost. However, this element is implicitly included along with the transaction costs in eq. (14), below.
} 
ponents of welfare cost are implicitly determined by the money-demand function of equations (3) and (4), which has the inflation rate elasticity as shown in equation (5). Since the private interest cost of holding money is $\left(r_{p}+r^{*}\right)(M / P)$, the imputed value of a marginal increment in real balances, $d(M / P)$, is $\left(r_{p}+r^{*}\right)$. In the current model this imputed value corresponds to the reduction in transaction costs of the two types mentioned above. Hence, the total transaction costs incurred at inflation rate $\bar{r}_{p}{ }^{17}$ is given by the integral which measures the area under the moneydemand curve: ${ }^{18}$

$$
W=\int_{\frac{M}{P}\left(\bar{r}_{p}+r^{*}\right)}^{\frac{M}{P}(0)}\left(r_{p}+r^{*}\right) d(M / P) .
$$

The integration can be carried out, using the money-demand function of equations (3) and (4), to yield a closed-form expression for $W$. The resulting expression is cumbersome and is not written out here. Since the integration under the money-demand curve in equation (14) implicitly includes the added transaction costs for movement into substitute payments media, the calculated value of $\mathrm{W}$ exceeds that indicated in equation (12). However, the departure from that equation is significant only when $r_{p}$ is substantially largee than $r^{*}$. The expression for welfare cost derived from equation (14) also retains the property that $W \rightarrow 0$ as $r_{p} \rightarrow-r^{*}$.

Values of $\mathrm{W}$ from equation (14), relative to real income $\mathrm{X}$, are tabulated in table 2 for various values of $r_{p}$ and for the two values of $r^{*}, 1 / 2$ percent and 1 percent per month. The numerical results are based on the overall estimate of $k$ and on the two overall estimates of $[A(Y / P)] / X$, which correspond to the two values of $r^{*}$, from table 1 . The calculations also assume no variation in $r^{*}$ as $r_{p}$ changes.

The estimated welfare cost is between 1 and 3 percent of income at $r_{p}=0$. Accordingly, these figures estimate the steady-state gain which would be obtained by moving from a zero rate of inflation to the optimum quantity of money where $r_{p}=-r^{*}$. It is interesting to compare these estimates with those obtained earlier by Friedman (1969, p. 44) for the United States. For an internal rate of discount (comparable with $r^{*}$ ) of 0.05 per year, Friedman estimates a potential welfare gain from moving to

17 The symbol $\bar{r}_{p}$ is used to distinguish the current rate of inflation from the running variable $r_{p}$ which appears inside the integral.

18 The approach of measuring social cost by integrating under the money-demand curve is discussed in Friedman (1953) and in Bailey (1956, pp. 102-3). This approach could also have been used when substitute transactions media were neglected to obtain equation (12). This earlier expression would emerge from the integration in eq. (14) if $k=0$ were set in eq. (4). 
the optimum quantity of money of $0.4-0.7$ percent of income, while for a rate of 0.33 per year he estimates a gain of 11.2-18.5 percent of income. Estimates of the welfare gain from my model are 1.2 percent and 7.8 percent of income for $r^{*}=.05$ per year and .33 per year, respectively. ${ }^{19}$ Hence, my results and Friedman's are of comparable magnitude, but mine are less sensitive to variations in $r^{*}$.

For rates of inflation between 2 and 5 percent per month, which are typical rates for some Latin American countries, the estimated welfare cost is between 3 and 7 percent of income. When the rate of inflation rises above 5 percent per month, the welfare cost advances rapidly. For the 25-50 percent per month range of inflation rates, which typifies hyperinflations, the estimated welfare cost is between 11 and 22 percent of income. Finally, when the inflation rate rises as high as 100-150 percent per month, which is the range in which hyperinflations have tended to become unstable (see Section II, above), the estimated welfare cost is between 22 and 38 percent of income.

The marginal effect of inflation on welfare cost can be determined from differentiation of the integral in equation (14) $\mathrm{as}^{20}$

$$
\frac{d W}{d r_{p}}=-\eta_{r_{p}}(M / P)\left(\frac{r_{p}+r^{*}}{r_{p}}\right) \text {. }
$$

Since government revenue is given by $G=r_{p}(M / P)$, the marginal collection cost is

$$
\mathrm{MCC}=d W / d G=\frac{-\eta_{r_{p}}}{1+\eta_{r_{p}}} \cdot\left(\frac{r_{p}+r^{*}}{r_{p}}\right),
$$

${ }^{19}$ Friedman's estimate corresponding to the discount rate of 0.33 per year also involves his assumption that the discount rate would fall to 0.17 per year when the optimum quantity of money was attained. In fact, the calculation of welfare gain should not be affected by changes in $r^{*}$ as $r_{p}$ is reduced from 0 to $-r^{*}$. The expression for welfare gain from moving from a zero inflation rate to the optimum quantity of money is

$$
\begin{aligned}
W\left(r_{p^{\prime}}=0\right)= & \int_{\frac{M}{P}\left(r^{*}\right)}^{\frac{M}{P}(0)}\left(r_{p}+r^{*}\right) d(M / P) \\
& =\int_{r^{*}\left(r_{p}=0\right)}^{0} \eta_{r_{p+r^{*}}}(M / P) d\left(r_{p}+r^{*}\right),
\end{aligned}
$$

where $\eta r_{p+r^{*}}$ is the elasticity of $M / P$ with respect to $r_{p}+r^{*}$. Variations in $r^{*}$ as $r_{p}$ varies do not change the value of the integral. Viewed in the context of my model, the welfare gain corresponds to the transaction costs at $r_{p}=0$, which is $A(Y / P) \sqrt{r^{*}}$, which depends only on $r^{*}\left(r_{p}=0\right)$ and not on $r^{*}\left(r_{p}=-r^{*}\right)$.

20 The differentiation is actually with respect to $\bar{r}_{p}$, which appears in the lower limit of integration in equation (14). 
where the inflation rate elasticity is given as a function or $r_{p}$ and $r^{*}$ in equation (5). For moderate values of $r_{p}\left(r_{p} \sim r^{*}\right)$, the expression given in equation (13) is a satisfactory approximation to equation (15). For values of $r_{p}$ much above $r^{*}$, the MCC as shown in equation (13) understates the cost shown in equation (15), since the latter expression includes the cost of movements into substitute transactions media.

Values of MCC are tabulated for various values of $r_{p}$ in table 2. The most important conclusion, which stems from the general property of the model which is most apparent in equation (13), is that the marginal collection cost exceeds 50 percent for all positive values of $r_{p} .{ }^{21}$ Hence, generating revenue by producing inflation can be socially desirable only if the marginal cost of alternative revenue-raising schemes exceeds 50 percent. Empirically, Bailey (1956, p. 108) suggests that 7 percent is a reasonable approximation for tax collection costs (including misallocation costs) in countries with poor administrative systems. However, since Bailey is apparently referring to average, rather than to marginal, collection costs, the appropriate figure may be substantially higher, such as 15-20 percent. Nevertheless, if the 50 percent MCC figure for inflationary finance is accepted as reasonably accurate, one cannot make a plausible case for inflationary finance on social welfare grounds. Superficially, raising revenue by printing money has appeal, particularly to underdeveloped countries, because of the low direct administrative cost. However, the indirect cost is sufficiently great that a poorly administered income or sales tax should be preferable from the standpoint of social welfare.

\section{Additional Empirical Results}

Table 3 contains empirical estimates of the parameters, $\left(\alpha_{1}, k, b\right)$, from the model of equations (6) and (7) for the five cases of hyperinflation. These estimates are subject to the constraint, $\hat{\alpha}_{2}=-1 / 2$. The point estimates for Austria, Germany, Hungary I, and Poland are basically similar to those reported in the earlier paper (Barro $1970 b$, p. 1255), in which $\hat{\alpha}_{2}$ was unconstrained. Unconstrained estimates for Hungary II, reported in table 4 , are also similar to the estimates with $\hat{\alpha}_{2}=-1 / 2$. However, with $\alpha_{2}$ fixed, the (conditional) confidence intervals for $\hat{k}$ are considerably narrower than before. Because $\alpha_{2}=-1 / 2$ was suggested by the underlying theory, and because this conjecture was borne out by the empirical results from the unconstrained regressions (see table 4 and Barro 1970b, p. 1256), it is thought that more efficient estimates of $k$ (and of $\alpha_{1}$ and $b$ ) are obtained by constraining $\hat{\alpha}_{2}=-1 / 2$. Therefore, the estimates of $k$ from table 3 were used in the analysis of Sections II and III, above.

21 This conclusion is strengthened with the presence of real growth (see n. 9, above). In this case, when $r_{p} \sim r^{*}$, the MCC is $\left(r_{p}+r^{*}\right) /\left(r_{p}+2 r^{*}-\rho\right)$, which exceeds the value shown in equation (13) when $\rho>0$. 


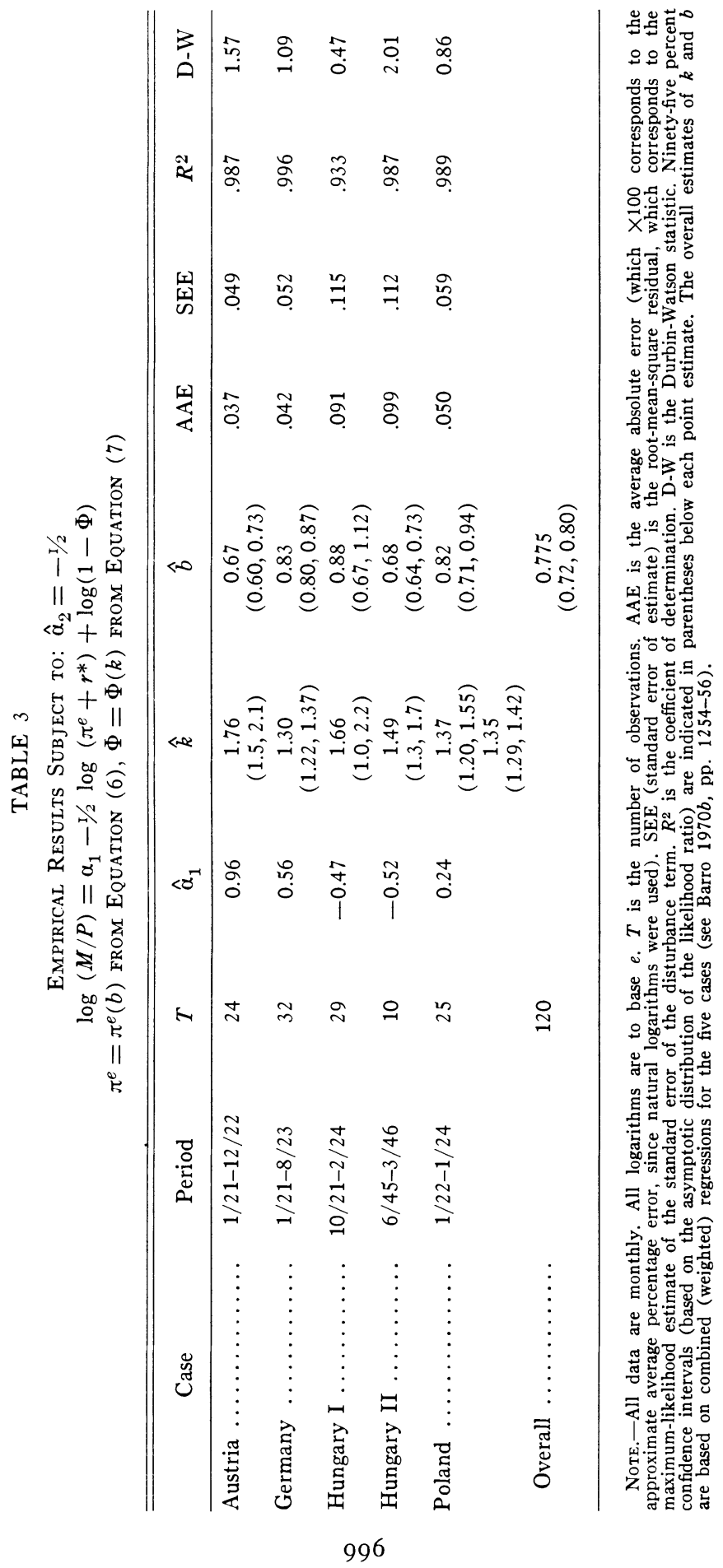




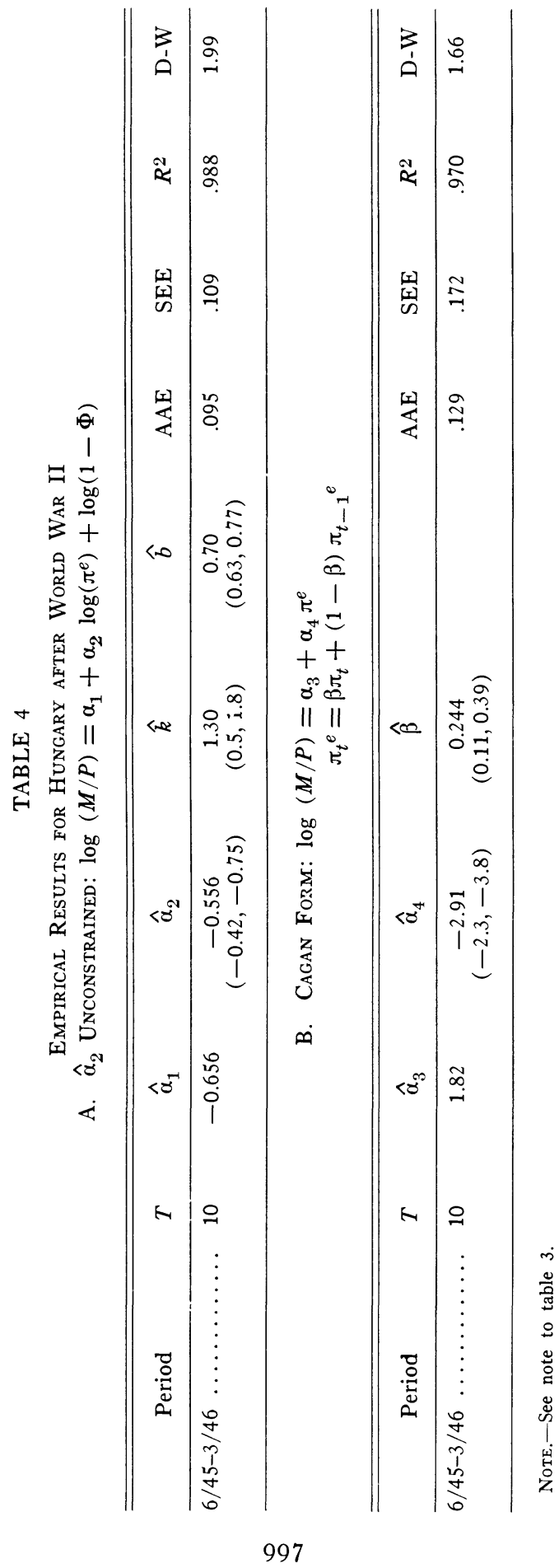


Table 2 (col. 6), above, contains values of $(M / P)\left(r_{p}\right) /(M / P)(0)$ for various values of $r_{p}$, corresponding to the overall estimate of $k$ from table 3 . The decline in $M / P$ with increasing $r_{p}$ is to 21-29 percent of $(M / P)$ (0) at $r_{p}=10$ percent per month, to $8-11$ percent of $(M / P)(0)$ at 50 percent per month, and to less than 1 percent of $(M / P)(0)$ at 500 percent per month. The decline in $M / P$ corresponds partly to a rise in velocity $[(1-\Phi)(Y / P) /(M / P)]$, as indicated in column 7 of table 2 , and partly to a fall in the fraction of monetized transactions $(1-\Phi)$. The effect of $r_{p}$ on $\Phi$ (col. 8) is small until extreme rates of inflation are attained. For example, only 4-5 percent of transactions have been "demonetized" at $\boldsymbol{r}_{p}$ $=10$ percent per month $-\mathrm{a}$ rate of inflation which is extremely high by conventional standards. Apparently, the convenience of money for making transactions is sufficiently great ( $k$ sufficiently low) that individuals are unwilling to abandon money as a means of payments for most transactions even at rates of inflation above a 100 percent annual rate. However, individuals do respond to an inflation rate of this magnitude by greatly speeding up the payments process - with velocity at $r_{p}=10$ percent per month three to five times as great as that at $r_{p}=0$. At $r_{p}=50$ percent per month, $\Phi$ is about 20 percent, and at $r_{p}=500$ percent per month, $\Phi$ is about 80 percent. Thus, at truly astronomical rates, individuals are willing to substitute alternative transactions media, but the substitution process is not complete even at the incredible inflation rate of 500 percent per month.

With the inclusion of results for the second Hungarian hyperinflation, it is possible to make an interesting comparison of this experience with that which occurred twenty years earlier in the same country. On the basis of his empirical results (using Cagan's estimates), Bailey (1956, p. 108) conducts a "comparison of Hungary after the second World War with Hungary after the first" and concludes: "the share of national income that the government could get at a given welfare cost . . was far smaller in the second hyperinflation than in the first. One possible explanation for this is that a second hyperinflation within a single generation found people far better prepared to reduce their real cash balances than they had been when they had no such previous experience."

In terms of the underlying model used in this paper, the long-term effect suggested by Bailey can be interpreted as shifts in the $k, b$, or $[A(Y / P)] /$ $X$ parameters. For example, if people became more adept at developing and using convenient alternative means of payments, this change would reduce the average cost of employing a substitute transactions medium and would therefore raise the $k$ parameter (see Barro 1970b, p. 1242, and Section I, above). However, the estimated $k$-values for Hungary I and Hungary II, contained in table 3, do not differ significantly. Another interpretation is that long-run experience induces individuals to adjust more quickly to changing rates of inflation-hence, $b$ in equation (7) would increase in the long run. From the underlying model (Barro 1970b, 
pp. 1245,1250 ), a shift in $b$ could reflect a decrease in the cost of instituting changes in a given pattern of transactions behavior-for example, a reduction in the cost of changing the payments period. However, the empirical results in table 3 do not support the conjecture that $b$ for Hungary II exceeds that for Hungary I. Another possibility for reducing money demand in the long run is a cut in the $[A(Y / P)] / X$ parameter, which is included in table 1 . For a given $(Y / P) / X$, a fall in $A$ would reflect a decrease in transaction costs associated with the use of the conventional money (for example, a decrease in the cost of making wage payments). The estimated $[A(Y / P)] / X$ parameters have unknown reliability, but the values for Hungary I and Hungary II in table 1 appear to be similar.

Given the similarity in the $k$ and $[A(Y / P) / X]$ parameters, the estimated values of $\mu^{*}$ and $g^{*}$ for the two cases in table 1 are very close. Therefore, the results suggest that no significant structural shifts occurred from the first to the second Hungarian experience. ${ }^{22}$ While it is theoretically possible that previous experience with hyperinflation will produce shifts in $k, b$, or $[A(Y / P) / X]$ - and therefore produce shifts in $\mu^{*}$ and $g^{*}$ - this possibility is not borne out by the empirical evidence on the Hungarian experiences.

\section{Test for Absence of Money Illusion}

Since the underlying theory is a theory of real demand for money, all empirical estimation has been carried out, thus far, with $\log (M / P)$ as the dependent variable. The theoretical absence of money illusion in the demand function can be tested by using $-\log (P)$ as the dependent variable (since $P$ is regarded as endogenous, with $M$ exogenous) and including $-\log M$ as an independent variable with an unrestricted coefficient:

$-\log P=\alpha_{1}+\alpha_{2} \log \left(\pi^{e}+r^{*}\right)+\log (1-\Phi)$

$$
+\alpha_{3} \cdot(-\log M) \text {. }
$$

Absence of money illusion corresponds to $\alpha_{3}=1$ in equation (16).

Point estimates of $\alpha_{3}$ and likelihood ratio statistics for the null hypothesis, $\alpha_{3}=1$, are contained in table 5 for the five cases of hyperinflation. The results strongly verify the absence of money illusion for Austria and Germany, with the point estimates of $\alpha_{3}, 0.995$ and 1.023, respectively, extremely close to 1.0. The null hypothesis, $\alpha_{3}=1$, is also accepted for

22 The two Hungarian cases are even similar in respect to average regression error -approximately 9 percent for the first case and 10 percent for the second (table 3 ). These values contrast with the range of 4-5 percent error obtained in the other three cases. This result may suggest that: (a) Hungarians are inherently unpredictable, or (b) Hungarians always generate poor data. No doubt other explanations can be offered. 


\section{TABLE 5}

Test for Absence of Money Illusion

$\left[-\log P=\alpha_{1}+\alpha_{2} \log \left(\pi^{e}\right)+\log (1-\Phi)+\alpha_{3}(-\log M)\right]$

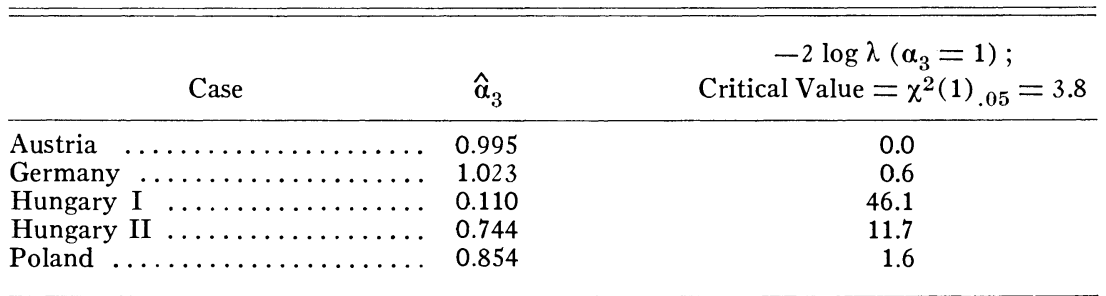

Note. $-2 \log \lambda=T \log \left(\mathrm{SSE}^{*} / \mathrm{SSE}\right)$, where $\lambda$ is the likelihood ratio. S $\hat{S E}$ is the minimum sum of squared errors with $\hat{a}_{3}$ unrestricted. $\mathrm{SSE}^{*}$ is the minimum subject to $\hat{a}_{3}=1$, and $T$ is the number of observations. Asymptotically, $-2 \log \lambda$ is distributed as $\chi^{2}(1)$ in this case.

Poland. The null hypothesis is rejected for both Hungarian cases. This rejection in the Hungarian cases is, in a sense, consistent with the large average errors that characterize the regression fits for these cases (table 3).$^{23}$ It is not possible to account for these problems at this point, though one possible source of difficulty is the assumed exogeneity of $M$ in equation (16). In any case, the satisfactory results on absence of money illusion for Austria, Germany, and Poland provide support for the underlying model.

23 The first Hungarian case also exhibits strong serial correlation of residuals (table 3), while the Polish and German cases exhibit smaller amounts of significant residual autocorrelation. The inclusion of some additional explanatory variables in the regression equations-a real-income proxy, a time-trend variable, and seasonal factors-did not remove the serial correlation in these cases (Barro 1970a, pp. 58-61). The four post-World War I cases have also been reestimated under the assumption that the error term was generated by a first-order Markov process: $u_{t}=\lambda u_{t-1}+v_{t}$ (Barro 1970a, pp. 62-66). Simultaneous estimation of $\lambda$ with the $\left(\alpha_{1}, \alpha_{2}, k, b\right)$ parameters showed that the previous estimates for Austria and Germany were insensitive to this shift in the assumed error process. The results for Poland were mixed, since the parameter estimates were not substantially altered by the inclusion of the $\lambda$-coefficient, but the first-order Markov process did not fully account for the residual autocorrelation in this case. The results for the first Hungarian case were entirely unsatisfactory, since the estimate of $\lambda$ did not converge below 1.0. This difficulty is consistent with the other problems that have been encountered in this case and suggests some type of specification error-that is, some error in the theoretical form or (more likely, considering the success in the other post-World War I cases) some data problems leading to serious errors in the measurement of variables. 


\section{Appendix}

Sample-Period Values for Hungary II, Based on Results Reported in TABle 4 ( $\hat{\alpha}_{2}$ Unconstrained)

\begin{tabular}{|c|c|c|c|c|c|c|}
\hline $\begin{array}{l}\text { End-of- } \\
\text { Month }\end{array}$ & $\beta_{t}$ & $\pi_{t}$ & $\sigma_{t}^{e}$ & $\begin{array}{l}\log (M / P)_{t} \\
\quad \text { (Actual) }\end{array}$ & $\begin{array}{l}\mathrm{og}(M / P)_{t} \\
\text { (Esti- } \\
\text { mated) }\end{array}$ & Residual \\
\hline \multicolumn{7}{|l|}{ 1945: } \\
\hline June $\ldots \ldots \ldots$ & 0.066 & 0.001 & 0.042 & 1.061 & 1.069 & -0.008 \\
\hline July $\ldots \ldots \ldots$ & 0.071 & 0.311 & 0.053 & 0.871 & 0.935 & -0.064 \\
\hline August $\ldots \ldots \ldots$ & 0.088 & 0.481 & 0.074 & 0.793 & 0.741 & 0.052 \\
\hline September .... & 0.117 & 0.795 & 0.118 & 0.564 & 0.451 & 0.112 \\
\hline October $\ldots \ldots$ & 0.232 & 1.867 & 0.338 & -0.339 & -0.247 & -0.092 \\
\hline November $\ldots .$. & 0.484 & 1.870 & 0.925 & -0.964 & -1.052 & 0.088 \\
\hline December $\quad \ldots \ldots$ & 0.636 & U.898 & 0.908 & -1.108 & -1.035 & -0.072 \\
\hline \multicolumn{7}{|l|}{ 1946: } \\
\hline January $\ldots . .$. & 0.597 & 0.656 & 0.752 & -0.995 & -0.871 & -0.124 \\
\hline February $\ldots .$. & 0.705 & 2.080 & 1.619 & -1.717 & -1.602 & -0.115 \\
\hline March $\ldots \ldots$ & 0.841 & 1.414 & 1.446 & -1.261 & -1.483 & 0.222 \\
\hline
\end{tabular}

Note.-Units for $\beta_{t}, \pi_{t}$, and $\pi_{t}{ }^{\theta}$ are per month (see eq. [6]). $M$ is an end-of-month measure of bank notes issued by the National Bank of Hungary in units of $10^{9}$ Pengos (U.N., Monthly BullStatis. [January 1947, June 1947]). $P$ is an end-of-month index of the cost of living in Budapest, based on $1929=0.1$. From June 1944 to July 1946 the data is from U.N., Monthly Bull. Statis. (June 1947), and suppl. (June 1948), p. 143. From December 1940 to March 1944, price data have been obtained from the following sources: Ann. Statis. Hongrois (1940), p. 113: ibid. (1941), p. 150; Magyar Statis. Szemle (1943), pp. 232-33: ibid. (1944), pp. 376-77; Internat. Labor Rev. (1944); and Wirtschaft und Statistik (1944). Unfortunately, it has not yet been possible to locate monthly data for the period April 1944-March 1945, and interpolation was necessary to generate $\pi_{t}$ for this period. If this data were located, it would be possible to increase the number of sample observations for Hungary II. The starting value for $\pi^{e}$ in January 1942 is taken to be 0.016 , the average rate of price change between December 1940 and December 1941.

\section{References}

Bailey, M. "The Welfare Cost of Inflationary Finance." J.P.E. 64 (April 1956): 93-110.

Barro, R. J. "Inflation, the Payments Period, and the Demand for Money." Ph.D. dissertation, Harvard Univ., 1970. (a)

$\rightarrow-$. "Inflation, the Payments Period, and the Demand for Money." J.P.E. 78 (November/December 1970): 1228-63. (b)

Cagan, P. "The Monetary Dynamics of Hyperinflation." In Studies in the Quantity Theory of Money, edited by M. Friedman. Chicago: Univ. Chicago Press, 1956.

Clower, R. W. "Introduction." In Monetary Theory, edited by R. W. Clower. Baltimore: Penguin, 1969.

Feige, E., and Parkin, M. "The Optimal Quantity of Money, Bonds, Commodity Inventories, and Capital." A.E.R. 61 (June 1971) : 335-49.

Friedman, M. "Discussion of the Inflationary Gap." Essays in Positive Economics. Chicago: Univ. Chicago Press, 1953.

- "The Optimum Quantity of Money." The Optimum Quantity of Money and Other Essays. Chicago: Aldine, 1969.

$\rightarrow-$. "Government Revenue from Inflation." J.P.E. 79 (July/August 1971): 846-56.

Santomero, A. M. "Optimal Transactions Behavior and the Demand for Money." Ph.D. dissertation, Brown Univ., 1971.

Stein, J. "Monetary Growth Theory in Perspective." A.E.R. 60 (March 1970): 85-106. 